Article

\title{
On $T$-Characterized Subgroups of Compact Abelian Groups
}

\section{Saak Gabriyelyan}

Department of Mathematics, Ben-Gurion University of the Negev, P.O. 653,

Beer-Sheva 8410501, Israel; E-Mail: saak@math.bgu.ac.il; Tel.: 972-8-646-7830

Academic Editor: Sidney A. Morris

Received: 16 February 2015 / Accepted: 16 June 2015 / Published: 19 June 2015

\begin{abstract}
A sequence $\left\{u_{n}\right\}_{n \in \omega}$ in abstract additively-written Abelian group $G$ is called a $T$-sequence if there is a Hausdorff group topology on $G$ relative to which $\lim _{n} u_{n}=0$. We say that a subgroup $H$ of an infinite compact Abelian group $X$ is $T$-characterized if there is a $T$-sequence $\mathbf{u}=\left\{u_{n}\right\}$ in the dual group of $X$, such that $H=\left\{x \in X:\left(u_{n}, x\right) \rightarrow 1\right\}$. We show that a closed subgroup $H$ of $X$ is $T$-characterized if and only if $H$ is a $G_{\delta}$-subgroup of $X$ and the annihilator of $H$ admits a Hausdorff minimally almost periodic group topology. All closed subgroups of an infinite compact Abelian group $X$ are $T$-characterized if and only if $X$ is metrizable and connected. We prove that every compact Abelian group $X$ of infinite exponent has a $T$-characterized subgroup, which is not an $F_{\sigma}$-subgroup of $X$, that gives a negative answer to Problem 3.3 in Dikranjan and Gabriyelyan (Topol. Appl. 2013, 160, 2427-2442).
\end{abstract}

Keywords: characterized subgroup; $T$-characterized subgroup; $T$-sequence; dual group; von Neumann radical

MSC classifications: 22A10; 43A40; 54H11

\section{Introduction}

Notation and preliminaries: Let $X$ be an Abelian topological group. We denote by $\widehat{X}$ the group of all continuous characters on $X$, and $\widehat{X}$ endowed with the compact-open topology is denoted by $X^{\wedge}$. The homomorphism $\alpha_{X}: X \rightarrow X^{\wedge \wedge}, x \mapsto(\chi \mapsto(\chi, x))$, is called the canonical homomorphism. Denote by $\mathbf{n}(X)=\cap_{\chi \in \hat{X}} \operatorname{ker}(\chi)=\operatorname{ker}\left(\alpha_{X}\right)$ the von Neumann radical of $X$. The group $X$ is called minimally almost periodic $(\operatorname{Min} A P)$ if $\mathbf{n}(X)=X$, and $X$ is called maximally almost periodic 
$(M A P)$ if $\mathbf{n}(X)=\{0\}$. Let $H$ be a subgroup of $X$. The annihilator of $H$ we denote by $H^{\perp}$, i.e., $H^{\perp}=\left\{\chi \in X^{\wedge}:(\chi, h)=1\right.$ for every $\left.h \in H\right\}$.

Recall that an Abelian group $G$ is of finite exponent or bounded if there exists a positive integer $n$, such that $n g=0$ for every $g \in G$. The minimal integer $n$ with this property is called the exponent of $G$ and is denoted by $\exp (G)$. When $G$ is not bounded, we write $\exp (G)=\infty$ and say that $G$ is of infinite exponent or unbounded. The direct sum of $\omega$ copies of an Abelian group $G$ we denote by $G^{(\omega)}$.

Let $\mathbf{u}=\left\{u_{n}\right\}_{n \in \omega}$ be a sequence in an Abelian group $G$. In general, no Hausdroff topology may exist in which $\mathbf{u}$ converges to zero. A very important question of whether there exists a Hausdorff group topology $\tau$ on $G$, such that $u_{n} \rightarrow 0$ in $(G, \tau)$, especially for the integers, has been studied by many authors; see Graev [1], Nienhuys [2], and others. Protasov and Zelenyuk [3] obtained a criterion that gives a complete answer to this question. Following [3], we say that a sequence $\mathbf{u}=\left\{u_{n}\right\}$ in an Abelian group $G$ is a $T$-sequence if there is a Hausdorff group topology on $G$ in which $u_{n}$ converges to zero. The finest group topology with this property we denote by $\tau_{\mathbf{u}}$.

The counterpart of the above question for precompact group topologies on $\mathbb{Z}$ is studied by Raczkowski [4]. Following [5,6] and motivated by [4], we say that a sequence $\mathbf{u}=\left\{u_{n}\right\}$ is a $T B$-sequence in an Abelian group $G$ if there is a precompact Hausdorff group topology on $G$ in which $u_{n}$ converges to zero. For a $T B$-sequence $\mathbf{u}$, we denote by $\tau_{b \mathbf{u}}$ the finest precompact group topology on $G$ in which u converges to zero. Clearly, every $T B$-sequence is a $T$-sequence, but in general, the converse assertion does not hold.

While it is quite hard to check whether a given sequence is a $T$-sequence (see, for example, [3,7-10]), the case of $T B$-sequences is much simpler. Let $X$ be an Abelian topological group and $\mathbf{u}=\left\{u_{n}\right\}$ be a sequence in its dual group $X^{\wedge}$. Following [11], set:

$$
s_{\mathbf{u}}(X)=\left\{x \in X:\left(u_{n}, x\right) \rightarrow 1\right\} .
$$

In [5], the following simple criterion to be a $T B$-sequence was obtained:

Fact 1 ([5]). A sequence $\mathbf{u}$ in a (discrete) Abelian group $G$ is a TB-sequence if and only if the subgroup $s_{\mathbf{u}}(X)$ of the (compact) dual $X=G^{\wedge}$ is dense.

Motivated by Fact 1, Dikranjan et al. [11] introduced the following notion related to subgroups of the form $s_{\mathbf{u}}(X)$ of a compact Abelian group $X$ :

Definition 2 ([11]). Let $H$ be a subgroup of a compact Abelian group $X$ and $\mathbf{u}=\left\{u_{n}\right\}$ be a sequence in $\widehat{X}$. If $H=s_{\mathbf{u}}(X)$, we say that $\mathbf{u}$ characterizes $H$ and that $H$ is characterized (by $\mathbf{u}$ ).

Note that for the torus $\mathbb{T}$, this notion was already defined in [12]. Characterized subgroups have been studied by many authors; see, for example, [11-16]. In particular, the main theorem of [15] (see also [14]) asserts that every countable subgroup of a compact metrizable Abelian group is characterized. It is natural to ask whether a closed subgroup of a compact Abelian group is characterized. The following easy criterion is given in [13]:

Fact 3 ([13]). A closed subgroup $H$ of a compact Abelian group $X$ is characterized if and only if $H$ is $a G_{\delta}$-subgroup. In particular, $X / H$ is metrizable, and the annihilator $H^{\perp}$ of $H$ is countable. 
The next fact follows easily from Definition 2:

Fact 4 ([17], see also [13]). Every characterized subgroup $H$ of a compact Abelian group $X$ is an $F_{\sigma \delta}$-subgroup of $X$, and hence, $H$ is a Borel subset of $X$.

Facts 3 and 4 inspired in [13] the study of the Borel hierarchy of characterized subgroups of compact Abelian groups. For a compact Abelian group $X$, denote by Char $(X)$ (respectively, $\mathrm{SF}_{\sigma}(X), \mathrm{SF}_{\sigma \delta}(X)$ and $\mathrm{SG}_{\delta}(X)$ ) the set of all characterized subgroups (respectively, $F_{\sigma}$-subgroups, $F_{\sigma \delta}$-subgroups and $G_{\delta}$-subgroups) of $X$. The next fact is Theorem E in [13]:

Fact 5 ([13]). For every infinite compact Abelian group X, the following inclusions hold:

$$
\mathrm{SG}_{\delta}(X) \varsubsetneqq \operatorname{Char}(X) \varsubsetneqq \mathrm{SF}_{\sigma \delta}(X) \text { and } \operatorname{SF}_{\sigma}(X) \nsubseteq \operatorname{Char}(X) \text {. }
$$

If in addition $X$ has finite exponent, then:

$$
\operatorname{Char}(X) \varsubsetneqq \mathrm{SF}_{\sigma}(X) .
$$

The inclusion Equation (1) inspired the following question:

Question 6 (Problem 3.3 in [13]). Does there exist a compact Abelian group X of infinite exponent all of whose characterized subgroups are $F_{\sigma}$-subsets of $X$ ?

Main results: It is important to emphasize that there is no restriction on the sequence $\mathbf{u}$ in Definition 2. If a characterized subgroup $H$ of a compact Abelian group $X$ is dense, then, by Fact 1 , a characterizing sequence is also a $T B$-sequence. However, if $H$ is not dense, we cannot expect in general that a characterizing sequence of $H$ is a $T$-sequence. Thus, it is natural to ask:

Question 7. For which characterized subgroups of compact Abelian groups can one find characterizing sequences that are also T-sequences?

This question is of independent interest, because every $T$-sequence $\mathbf{u}$ naturally defines the group topology $\tau_{\mathbf{u}}$ satisfying the following dual property:

Fact 8 ([18]). Let $H$ be a subgroup of an infinite compact Abelian group $X$ characterized by $a$ T-sequence $\mathbf{u}$. Then, $\left(\widehat{X}, \tau_{\mathbf{u}}\right)^{\wedge}=H\left(=s_{\mathbf{u}}(X)\right)$ and $\mathbf{n}\left(\widehat{X}, \tau_{\mathbf{u}}\right)=H^{\perp}$ algebraically.

This motivates us to introduce the following notion:

Definition 9. Let $H$ be a subgroup of a compact Abelian group $X$. We say that $H$ is a $T$-characterized subgroup of $X$ if there exists a T-sequence $\mathbf{u}=\left\{u_{n}\right\}_{n \in \omega}$ in $\widehat{X}$, such that $H=s_{\mathbf{u}}(X)$.

Denote by $\operatorname{Char}_{T}(X)$ the set of all $T$-characterized subgroups of a compact Abelian group $X$. Clearly, $\operatorname{Char}_{T}(X) \subseteq$ Char $(X)$. Hence, if a $T$-characterized subgroup $H$ of $X$ is closed, it is a $G_{\delta}$-subgroup of $X$ by Fact 3 . Note also that $X$ is $T$-characterized by the zero sequence.

The main goal of the article is to obtain a complete description of closed $T$-characterized subgroups (see Theorem 10) and to study the Borel hierarchy of $T$-characterized subgroups (see Theorem 18) 
of compact Abelian groups. In particular, we obtain a complete answer to Question 7 for closed characterized subgroups and give a negative answer to Question 6.

Note that, if a compact Abelian group $X$ is finite, then every $T$-sequence $\mathbf{u}$ in $\widehat{X}$ is eventually equal to zero. Hence, $s_{\mathbf{u}}(X)=X$. Thus, $X$ is the unique $T$-characterized subgroup of $X$. Therefore, in what follows, we shall consider only infinite compact groups.

The following theorem describes all closed subgroups of compact Abelian groups that are $T$-characterized.

Theorem 10. Let $H$ be a proper closed subgroup of an infinite compact Abelian group $X$. Then, the following assertions are equivalent:

(1) $H$ is a $T$-characterized subgroup of $X$;

(2) $H$ is a $G_{\delta}$-subgroup of $X$, and the countable group $H^{\perp}$ admits a Hausdorff MinAPgroup topology;

(3) $H$ is a $G_{\delta}$-subgroup of $X$ and one of the following holds:

(a) $H^{\perp}$ has infinite exponent;

(b) $H^{\perp}$ has finite exponent and contains a subgroup that is isomorphic to $\mathbb{Z}\left(\exp \left(H^{\perp}\right)\right)^{(\omega)}$.

Corollary 11. Let $X$ be an infinite compact metrizable Abelian group. Then, the trivial subgroup $H=\{0\}$ is T-characterized if and only if $\widehat{X}$ admits a Hausdorff MinAP group topology.

As an immediate corollary of Fact 3 and Theorem 10, we obtain a complete answer to Question 7 for closed characterized subgroups.

Corollary 12. A proper closed characterized subgroup $H$ of an infinite compact Abelian group $X$ is T-characterized if and only if $H^{\perp}$ admits a Hausdorff MinAP group topology.

If $H$ is an open proper subgroup of $X$, then $H^{\perp}$ is non-trivial and finite. Thus, every Hausdorff group topology on $H^{\perp}$ is discrete. Taking into account Fact 3, we obtain:

Corollary 13. Every open proper subgroup $H$ of an infinite compact Abelian group $X$ is a characterized non-T-characterized subgroup of $X$.

Nevertheless (see Example 1 below), there is a compact metrizable Abelian group $X$ with a countable $T$-characterized subgroup $H$, such that its closure $\bar{H}$ is open. Thus, it may happen that the closure of a $T$-characterized subgroup is not $T$-characterized.

It is natural to ask for which compact Abelian groups all of their closed $G_{\delta}$-subgroups are $T$-characterized. The next theorem gives a complete answer to this question.

Theorem 14. Let $X$ be an infinite compact Abelian group. The following assertions are equivalent:

(1) All closed $G_{\delta^{-}}$-subgroups of $X$ are $T$-characterized;

(2) $X$ is connected.

By Corollary 2.8 of [13], the trivial subgroup $H=\{0\}$ of a compact Abelian group $X$ is a $G_{\delta}$-subgroup if and only if $X$ is metrizable. Therefore, we obtain: 
Corollary 15. All closed subgroups of an infinite compact Abelian group $X$ are T-characterized if and only if $X$ is metrizable and connected.

Theorems 10 and 14 are proven in Section 2.

In the next theorem, we give a negative answer to Question 6:

Theorem 16. Every compact Abelian group of infinite exponent has a dense T-characterized subgroup, which is not an $F_{\sigma}$-subgroup.

As a corollary of the inclusion Equation (1) and Theorem 16, we obtain:

Corollary 17. For an infinite compact Abelian group $X$, the following assertions are equivalent:

(i) X has finite exponent;

(ii) every characterized subgroup of $X$ is an $F_{\sigma}$-subgroup;

(iii) every $T$-characterized subgroup of $X$ is an $F_{\sigma}$-subgroup.

Therefore, $\operatorname{Char}(X) \subseteq \mathrm{SF}_{\sigma}(X)$ if and only if $X$ has finite exponent.

In the next theorem, we summarize the obtained results about the Borel hierarchy of $T$-characterized subgroups of compact Abelian groups.

Theorem 18. Let $X$ be an infinite compact Abelian group $X$. Then:

(1) $\operatorname{Char}_{T}(X) \varsubsetneqq \mathrm{SF}_{\sigma \delta}(X)$;

(2) $\mathrm{SG}_{\delta}(X) \bigcap \operatorname{Char}_{T}(X) \varsubsetneqq \operatorname{Char}_{T}(X)$;

(3) $\mathrm{SG}_{\delta}(X) \subseteq \operatorname{Char}_{T}(X)$ if and only if $X$ is connected;

(4) $\operatorname{Char}_{T}(X) \cap \mathrm{SF}_{\sigma}(X) \varsubsetneqq \mathrm{SF}_{\sigma}(X)$;

(5) $\operatorname{Char}_{T}(X) \subseteq \mathrm{SF}_{\sigma}(X)$ if and only if $X$ has finite exponent.

We prove Theorems 16 and 18 in Section 3.

The notions of $\mathfrak{g}$-closed and $\mathfrak{g}$-dense subgroups of a compact Abelian group $X$ were defined in [11]. In the last section of the paper, in analogy to these notions, we define $\mathfrak{g}_{T}$-closed and $\mathfrak{g}_{T}$-dense subgroups of $X$. In particular, we show that every $\mathfrak{g}_{T}$-dense subgroup of a compact Abelian group $X$ is dense if and only if $X$ is connected (see Theorem 37 ).

\section{The Proofs of Theorems 10 and 14}

The subgroup of a group $G$ generated by a subset $A$ we denote by $\langle A\rangle$.

Recall that a subgroup $H$ of an Abelian topological group $X$ is called dually closed in $X$ if for every $x \in X \backslash H$, there exists a character $\chi \in H^{\perp}$, such that $(\chi, x) \neq 1 . H$ is called dually embedded in $X$ if every character of $H$ can be extended to a character of $X$. Every open subgroup of $X$ is dually closed and dually embedded in $X$ by Lemma 3 of [19].

The next notion generalizes the notion of the maximal extension in the class of all compact Abelian groups introduced in [20]. 
Definition 19. Let $\mathcal{G}$ be an arbitrary class of topological groups. Let $(G, \tau) \in \mathcal{G}$ and $H$ be a subgroup of $G$. The group $(G, \tau)$ is called a maximal extension of $\left(H,\left.\tau\right|_{H}\right)$ in the class $\mathcal{G}$ if $\sigma \leq \tau$ for every group topology on $G$, such that $\left.\sigma\right|_{H}=\left.\tau\right|_{H}$ and $(G, \sigma) \in \mathcal{G}$.

Clearly, the maximal extension is unique if it exists. Note that in Definition 19, we do not assume that $\left(H,\left.\tau\right|_{H}\right)$ belongs to the class $\mathcal{G}$.

If $H$ is a subgroup of an Abelian group $G$ and $\mathbf{u}$ is a $T$-sequence (respectively, a $T B$-sequence) in $H$, we denote by $\tau_{\mathbf{u}}(H)$ (respectively, $\tau_{b \mathbf{u}}(H)$ ) the finest (respectively, precompact) group topology on $H$ generated by $\mathbf{u}$. We use the following easy corollary of the definition of $T$-sequences.

Lemma 20. For a sequence $\mathbf{u}$ in an Abelian group $G$, the following assertions are equivalent:

(1) $\mathbf{u}$ is a $T$-sequence in $G$;

(2) $\mathbf{u}$ is a $T$-sequence in every subgroup of $G$ containing $\langle\mathbf{u}\rangle$;

(3) $\mathbf{u}$ is a $T$-sequence in $\langle\mathbf{u}\rangle$.

In this case, $\langle\mathbf{u}\rangle$ is open in $\tau_{\mathbf{u}}$ (and hence, $\langle\mathbf{u}\rangle$ is dually closed and dually embedded in $\left(G, \tau_{\mathbf{u}}\right)$ ), and $\left(G, \tau_{\mathbf{u}}\right)$ is the maximal extension of $\left(\langle\mathbf{u}\rangle, \tau_{\mathbf{u}}(\langle\mathbf{u}\rangle)\right.$ in the class TAG of all Abelian topological groups.

Proof. Evidently, (1) implies (2) and (2) implies (3). Let $\mathbf{u}$ be a $T$-sequence in $\langle\mathbf{u}\rangle$. Let $\tau$ be the topology on $G$ whose base is all translationsof $\tau_{\mathbf{u}}(\langle\mathbf{u}\rangle)$-open sets. Clearly, $\mathbf{u}$ converges to zero in $\tau$. Thus, $\mathbf{u}$ is a $T$-sequence in $G$. Therefore, (3) implies (1).

Let us prove the last assertion. By the definition of $\tau_{\mathbf{u}}$, we have also $\tau \leq \tau_{\mathbf{u}}$, and hence, $\left.\tau\right|_{\langle\mathbf{u}\rangle}=\tau_{\mathbf{u}}(\langle\mathbf{u}\rangle) \leq\left.\tau_{\mathbf{u}}\right|_{\langle\mathbf{u}\rangle}$. Thus, $\langle\mathbf{u}\rangle$ is open in $\tau_{\mathbf{u}}$, and hence, it is dually closed and dually embedded in $\left(G, \tau_{\mathbf{u}}\right)$ by [19] (Lemma 3.3). On the other hand, $\left.\tau_{\mathbf{u}}\right|_{\langle\mathbf{u}\rangle} \leq \tau_{\mathbf{u}}(\langle\mathbf{u}\rangle)=\left.\tau\right|_{\langle\mathbf{u}\rangle}$ by the definition of $\tau_{\mathbf{u}}(\langle\mathbf{u}\rangle)$. Therefore, $\tau_{\mathbf{u}}$ is an extension of $\tau_{\mathbf{u}}(\langle\mathbf{u}\rangle)$. Now, clearly, $\tau=\tau_{\mathbf{u}}$, and $\left(G, \tau_{\mathbf{u}}\right)$ is the maximal extension of $\left(\langle\mathbf{u}\rangle, \tau_{\mathbf{u}}(\langle\mathbf{u}\rangle)\right.$ in the class TAG.

For $T B$-sequences, we have the following:

Lemma 21. For a sequence $\mathbf{u}$ in an Abelian group $G$, the following assertions are equivalent:

(1) $\mathbf{u}$ is a TB-sequence in $G$;

(2) $\mathbf{u}$ is a TB-sequence in every subgroup of $G$ containing $\langle\mathbf{u}\rangle$;

(3) $\mathbf{u}$ is a TB-sequence in $\langle\mathbf{u}\rangle$.

In this case, the subgroup $\langle\mathbf{u}\rangle$ is dually closed and dually embedded in $\left(G, \tau_{b \mathbf{u}}\right)$, and $\left(G, \tau_{b \mathbf{u}}\right)$ is the maximal extension of $\left(\langle\mathbf{u}\rangle, \tau_{b \mathbf{u}}(\langle\mathbf{u}\rangle)\right)$ in the class of all precompact Abelian groups.

Proof. Evidently, (1) implies (2) and (2) implies (3). Let $\mathbf{u}$ be a $T B$-sequence in $\langle\mathbf{u}\rangle$. Then, $\left(\langle\mathbf{u}\rangle, \tau_{b \mathbf{u}}(\langle\mathbf{u}\rangle)\right)^{\wedge}$ separates the points of $\langle\mathbf{u}\rangle$. Let $\tau$ be the topology on $G$ whose base is all translations of $\tau_{b \mathbf{u}}(\langle\mathbf{u}\rangle)$-open sets. Then, $\left(\langle\mathbf{u}\rangle, \tau_{b \mathbf{u}}(\langle\mathbf{u}\rangle)\right)$ is an open subgroup of $(G, \tau)$. It is easy to see that $(G, \tau)^{\wedge}$ separates the points of $G$. Since $\mathbf{u}$ converges to zero in $\tau$, it also converges to zero in $\tau^{+}$, where $\tau^{+}$is the Bohr topology of $(G, \tau)$. Thus, $\mathbf{u}$ is a $T B$-sequence in $G$. Therefore, (3) implies (1).

The last assertion follows from Proposition 1.8 and Lemma 3.6 in [20]. 
For a sequence $\mathbf{u}=\left\{u_{n}\right\}_{n \in \omega}$ of characters of a compact Abelian group $X$, set:

$$
K_{\mathbf{u}}=\bigcap_{n \in \omega} \operatorname{ker}\left(u_{n}\right)
$$

The following assertions is proven in [13]:

Fact 22 (Lemma 2.2(i) of [13]). For every sequence $\mathbf{u}=\left\{u_{n}\right\}_{n \in \omega}$ of characters of a compact Abelian group $X$, the subgroup $K_{\mathbf{u}}$ is a closed $G_{\delta}$-subgroup of $X$ and $K_{\mathbf{u}}=\langle\mathbf{u}\rangle^{\perp}$.

The next two lemmas are natural analogues of Lemmas 2.2(ii) and 2.6 of [13].

Lemma 23. Let $X$ be a compact Abelian group and $\mathbf{u}=\left\{u_{n}\right\}_{n \in \omega}$ be a T-sequence in $\widehat{X}$. Then, $s_{\mathbf{u}}(X) / K_{\mathbf{u}}$ is a T-characterized subgroup of $X / K_{\mathbf{u}}$.

Proof. Set $H:=s_{\mathbf{u}}(X)$ and $K:=K_{\mathbf{u}}$. Let $q: X \rightarrow X / K$ be the quotient map. Then, the adjoint homomorphism $q^{\wedge}$ is an isomorphism from $(X / K)^{\wedge}$ onto $K^{\perp}$ in $X^{\wedge}$. For every $n \in \omega$, define the character $\widetilde{u}_{n}$ of $X / K$ as follows: $\left(\widetilde{u}_{n}, q(x)\right)=\left(u_{n}, x\right)\left(\widetilde{u}_{n}\right.$ is well-defined, since $\left.K \subseteq \operatorname{ker}\left(u_{n}\right)\right)$. Then, $\widetilde{\mathbf{u}}=\left\{\widetilde{u}_{n}\right\}_{n \in \omega}$ is a sequence of characters of $X / K$, such that $q^{\wedge}\left(\widetilde{u}_{n}\right)=u_{n}$. Since $\mathbf{u} \subset K^{\perp}, \mathbf{u}$ is a $T$-sequence in $K^{\perp}$ by Lemma 20. Hence, $\widetilde{\mathbf{u}}$ is a $T$-sequence in $(X / K)^{\wedge}$ because $q^{\wedge}$ is an isomorphism.

We claim that $H / K=s_{\widetilde{\mathbf{u}}}(X / K)$. Indeed, for every $h+K \in H / K$, by definition, we have $\left(\widetilde{u}_{n}, h+K\right)=\left(u_{n}, h\right) \rightarrow 1$. Thus, $H / K \subseteq s_{\widetilde{\mathbf{u}}}(X / K)$. If $x+K \in s_{\widetilde{\mathbf{u}}}(X / K)$, then $\left(\widetilde{u}_{n}, x+K\right)=$ $\left(u_{n}, x\right) \rightarrow 1$. This yields $x \in H$. Thus, $x+K \in H / K$.

Let $\mathbf{u}=\left\{u_{n}\right\}_{n \in \omega}$ be a $T$-sequence in an Abelian group $G$. For every natural number $m$, set $\mathbf{u}_{m}=\left\{u_{n}\right\}_{n \geq m}$. Clearly, $\mathbf{u}_{m}$ is a $T$-sequence in $G, \tau_{\mathbf{u}}=\tau_{\mathbf{u}_{m}}$ and $s_{\mathbf{u}}(X)=s_{\mathbf{u}_{m}}(X)$ for every natural number $m$.

Lemma 24. Let $K$ be a closed subgroup of a compact Abelian group $X$ and $q: X \rightarrow X / K$ be the quotient map. Then, $\widetilde{H}$ is a $T$-characterized subgroup of $X / K$ if and only if $q^{-1}(\widetilde{H})$ is a $T$-characterized subgroup of $X$.

Proof. Let $\widetilde{H}$ be a $T$-characterized subgroup of $X / K$, and let a $T$-sequence $\widetilde{\mathbf{u}}=\left\{\widetilde{u}_{n}\right\}_{n \in \omega^{-}}$characterized $\widetilde{H}$. Set $H:=q^{-1}(\widetilde{H})$. We have to show that $H$ is a $T$-characterized subgroup of $X$.

Note that the adjoint homomorphism $q^{\wedge}$ is an isomorphism from $(X / K)^{\wedge}$ onto $K^{\perp}$ in $X^{\wedge}$. Set $\mathbf{u}=\left\{u_{n}\right\}_{n \in \omega}$, where $u_{n}=q^{\wedge}\left(\widetilde{u}_{n}\right)$. Since $q^{\wedge}$ is injective, $\mathbf{u}$ is a $T$-sequence in $K^{\perp}$. By Lemma 20, $\mathbf{u}$ is a $T$-sequence in $\widehat{X}$. Therefore, it is enough to show that $H=s_{\mathbf{u}}(X)$. This follows from the following chain of equivalences. By definition, $x \in s_{\mathbf{u}}(X)$ if and only if:

$$
\left(u_{n}, x\right) \rightarrow 1 \Leftrightarrow\left(\widetilde{u}_{n}, q(x)\right) \rightarrow 1 \Leftrightarrow q(x) \in \widetilde{H}=H / K \Leftrightarrow x \in H .
$$

The last equivalence is due to the inclusion $K \subseteq H$.

Conversely, let $H:=q^{-1}(\widetilde{H})$ be a $T$-characterized subgroup of $X$ and a $T$-sequence $\mathbf{u}=\left\{u_{n}\right\}_{n \in \omega}$-characterized $H$. Proposition 2.5 of [13] implies that we can find $m \in \mathbb{N}$, such that $K \subseteq K_{\mathbf{u}_{m}}$. Therefore, taking into account that $H=s_{\mathbf{u}}(X)=s_{\mathbf{u}_{m}}(X)$ for every natural 
number $m$, without loss of generality, we can assume that $K \subseteq K_{\mathbf{u}}$. By Lemma 23, $H / K_{\mathbf{u}}$ is a $T$-characterized subgroup of $X / K_{\mathbf{u}}$. Denote by $q_{u}$ the quotient homomorphism from $X / K$ onto $X / K_{\mathbf{u}}$. Then, $\widetilde{H}=q_{u}^{-1}\left(H / K_{\mathbf{u}}\right)$ is $T$-characterized in $X / K$ by the previous paragraph of the proof.

The next theorem is an analogue of Theorem B of [13], and it reduces the study of $T$-characterized subgroups of compact Abelian groups to the study of $T$-characterized ones of compact Abelian metrizable groups:

Theorem 25. A subgroup $H$ of a compact Abelian group $X$ is $T$-characterized if and only if $H$ contains a closed $G_{\delta}$-subgroup $K$ of $X$, such that $H / K$ is a $T$-characterized subgroup of the compact metrizable group $X / K$.

Proof. Let $H$ be $T$-characterized in $X$ by a $T$-sequence $\mathbf{u}=\left\{u_{n}\right\}_{n \in \omega}$ in $\widehat{X}$. Set $K:=K_{\mathbf{u}}$. Since $K$ is a closed $G_{\delta}$-subgroup of $X$ by Fact $22, X / K$ is metrizable. By Lemma $23, H / K$ is a $T$-characterized subgroup of $X / K$.

Conversely, let $H$ contain a closed $G_{\delta}$-subgroup $K$ of $X$, such that $H / K$ is a $T$-characterized subgroup of the compact metrizable group $X / K$. Then, $H$ is a $T$-characterized subgroup of $X$ by Lemma 24.

As was noticed in [21] before Definition 2.33, for every $T$-sequence $\mathbf{u}$ in an infinite Abelian group $G$, the subgroup $\langle\mathbf{u}\rangle$ is open in $\left(G, \tau_{\mathbf{u}}\right)$ (see also Lemma 20), and hence, by Lemmas 1.4 and 2.2 of [22], the following sequences are exact:

$$
\begin{aligned}
& 0 \rightarrow\left(\langle\mathbf{u}\rangle, \tau_{\mathbf{u}}\right) \rightarrow\left(G, \tau_{\mathbf{u}}\right) \rightarrow G /\langle\mathbf{u}\rangle \rightarrow 0 \\
& 0 \rightarrow(G /\langle\mathbf{u}\rangle)^{\wedge} \rightarrow\left(G, \tau_{\mathbf{u}}\right)^{\wedge} \rightarrow\left(\langle\mathbf{u}\rangle, \tau_{\mathbf{u}} \mid\langle\mathbf{u}\rangle\right)^{\wedge} \rightarrow 0
\end{aligned}
$$

where $(G /\langle\mathbf{u}\rangle)^{\wedge} \cong\langle\mathbf{u}\rangle^{\perp}$ is a compact subgroup of $\left(G, \tau_{\mathbf{u}}\right)^{\wedge}$ and $\left(\langle\mathbf{u}\rangle, \tau_{\mathbf{u}}\right)^{\wedge} \cong\left(G, \tau_{\mathbf{u}}\right)^{\wedge} /\langle\mathbf{u}\rangle^{\perp}$.

Let $\mathbf{u}=\left\{u_{n}\right\}_{n \in \omega}$ be a $T$-sequence in an Abelian group $G$. It is known [10] that $\tau_{\mathbf{u}}$ is sequential, and hence, $\left(G, \tau_{\mathbf{u}}\right)$ is a $k$-space. Therefore, the natural homomorphism $\alpha:=\alpha_{\left(G, \tau_{\mathbf{u}}\right)}:\left(G, \tau_{\mathbf{u}}\right) \rightarrow\left(G, \tau_{\mathbf{u}}\right)^{\wedge \wedge}$ is continuous by [23] (5.12). Let us recall that $\left(G, \tau_{\mathbf{u}}\right)$ is MinAP if and only if $\left(G, \tau_{\mathbf{u}}\right)=\operatorname{ker}(\alpha)$.

To prove Theorem 10, we need the following:

Fact 26 ([16]). For each T-sequence $\mathbf{u}$ in a countably infinite Abelian group $G$, the group $\left(G, \tau_{\mathbf{u}}\right)^{\wedge}$ is Polish.

Now, we are in a position to prove Theorem 10.

Proof of Theorem 10. (1) $\Rightarrow(2)$ Let $H$ be a proper closed $T$-characterized subgroup of $X$ and a $T$-sequence $\mathbf{u}=\left\{u_{n}\right\}_{n \in \omega^{-}}$-characterized $H$. Since $H$ is also characterized, it is a $G_{\delta^{-}}$subgroup of $X$ by Fact 3 . We have to show that $H^{\perp}$ admits a MinAP group topology.

Our idea of the proof is the following. Set $G:=\widehat{X}$. By Fact $8, H^{\perp}$ is the von Neumann radical of $\left(G, \tau_{\mathbf{u}}\right)$. Now, assume that we found another $T$-sequence $\mathbf{v}$ that characterizes $H$ and such that $\langle\mathbf{v}\rangle=H^{\perp}$ (maybe $\mathbf{v}=\mathbf{u}$ ). By Fact 8 , we have $\mathbf{n}\left(G, \tau_{\mathbf{v}}\right)=H^{\perp}=\langle\mathbf{v}\rangle$. Lemma 20 implies that the subgroup $\left(\langle\mathbf{v}\rangle,\left.\tau_{\mathbf{v}}\right|_{\langle\mathbf{v}\rangle}\right)$ of $\left(G, \tau_{\mathbf{v}}\right)$ is open, and hence, it is dually closed and dually embedded in $\left(G, \tau_{\mathbf{v}}\right)$. 
Hence, $\mathbf{n}\left(\langle\mathbf{v}\rangle,\left.\tau_{\mathbf{v}}\right|_{\langle\mathbf{v}\rangle}\right)=\mathbf{n}\left(G, \tau_{\mathbf{v}}\right)(=\langle\mathbf{v}\rangle)$ by Lemma 4 of [16]. Therefore, $\left(\langle\mathbf{v}\rangle,\left.\tau_{\mathbf{v}}\right|_{\langle\mathbf{v}\rangle}\right)$ is MinAP. Thus, $H^{\perp}=\langle\mathbf{v}\rangle$ admits a MinAP group topology, as desired.

We find such a $T$-sequence $\mathbf{v}$ in four steps (in fact, we show that $\mathbf{v}$ has the form $\mathbf{u}_{m}$ for some $m \in \mathbb{N}$ ).

Step 1. Let $q: X \rightarrow X / K_{\mathbf{u}}$ be the quotient map. For every $n \in \omega$, define the character $\widetilde{u}_{n}$ of $X / K_{\mathbf{u}}$ by the equality $u_{n}=\widetilde{u}_{n} \circ q$ (this is possible since $K_{\mathbf{u}} \subseteq \operatorname{ker}\left(u_{n}\right)$ ). As was shown in the proof of Lemma 23, the sequence $\widetilde{\mathbf{u}}=\left\{\widetilde{u}_{n}\right\}_{n \in \omega}$ is a $T$-sequence, which characterizes $H / K_{\mathbf{u}}$ in $X / K_{\mathbf{u}}$. Set $\widetilde{X}:=X / K_{\mathbf{u}}$ and $\widetilde{H}:=H / K_{\mathbf{u}}$. Therefore, $\widetilde{H}=s_{\widetilde{\mathbf{u}}}(\widetilde{X})$. By [24] (5.34 and 24.11) and since $K_{\mathbf{u}} \subseteq H$, we have:

$$
H^{\perp} \cong(X / H)^{\wedge} \cong(\widetilde{X} / \widetilde{H})^{\wedge} \cong \widetilde{H}^{\perp}
$$

By Fact 3, $\widetilde{X}$ is metrizable. Hence, $\widetilde{H}$ is also compact and metrizable, and $\widetilde{G}:=\widehat{\widetilde{X}}$ is a countable Abelian group by [24] (24.15). Since $H$ is a proper closed subgroup of $X$, Equation (3) implies that $\widetilde{G}$ is non-zero.

We claim that $\widetilde{G}$ is countably infinite. Indeed, suppose for a contradiction that $\widetilde{G}$ is finite. Then, $X / K_{\mathbf{u}}=\widetilde{X}$ is also finite. Now, Fact 22 implies that $\langle\mathbf{u}\rangle$ is a finite subgroup of $G$. Since $\mathbf{u}$ is a $T$-sequence, $\mathbf{u}$ must be eventually equal to zero. Hence, $H=s_{\mathbf{u}}(X)=X$ is not a proper subgroup of $X$, a contradiction.

Step 2. We claim that there is a natural number $m$, such that the group $\left(\left\langle\widetilde{\mathbf{u}}_{m}\right\rangle,\left.\tau_{\widetilde{\mathbf{u}}}\right|_{\left\langle\widetilde{\mathbf{u}}_{m}\right\rangle}\right)=$ $\left(\left\langle\widetilde{\mathbf{u}}_{m}\right\rangle,\left.\tau_{\widetilde{\mathbf{u}}_{m}}\right|_{\left\langle\widetilde{\mathbf{u}}_{m}\right\rangle}\right)$ is MinAP.

Indeed, since $\widetilde{G}$ is countably infinite, we can apply Fact 8 . Therefore, $\widetilde{H}=\left(\widetilde{G}, \tau_{\widetilde{\mathbf{u}}}\right)^{\wedge}$ algebraically. Since $\widetilde{H}$ and $\left(\widetilde{G}, \tau_{\widetilde{\mathbf{u}}}\right)^{\wedge}$ are Polish groups (see Fact 26$), \widetilde{H}$ and $\left(\widetilde{G}, \tau_{\widetilde{\mathbf{u}}}\right)^{\wedge}$ are topologically isomorphic by the uniqueness of the Polish group topology. Hence $\left(\widetilde{G}, \tau_{\widetilde{\mathbf{u}}}\right)^{\wedge \wedge}=\widetilde{H}^{\wedge}$ is discrete. As was noticed before the proof, the natural homomorphism $\widetilde{\alpha}:\left(\widetilde{G}, \tau_{\widetilde{\mathbf{u}}}\right) \rightarrow\left(\widetilde{G}, \tau_{\widetilde{\mathbf{u}}}\right)^{\wedge \wedge}$ is continuous. Since $\left(\widetilde{G}, \tau_{\widetilde{\mathbf{u}}}\right)^{\wedge \wedge}$ is discrete, we obtain that the von Neumann radical $\operatorname{ker}(\widetilde{\alpha})$ of $\left(\widetilde{G}, \tau_{\widetilde{\mathbf{u}}}\right)$ is open in $\tau_{\widetilde{\mathbf{u}}}$. Therefore, there exists a natural number $m$, such that $\widetilde{u}_{n} \in \operatorname{ker}(\widetilde{\alpha})$ for every $n \geq m$. Hence, $\left\langle\widetilde{\mathbf{u}}_{m}\right\rangle \subseteq \operatorname{ker}(\widetilde{\alpha})$. Lemma 20 implies that the subgroup $\left\langle\widetilde{\mathbf{u}}_{m}\right\rangle$ is open in $\left(\widetilde{G}, \tau_{\widetilde{\mathbf{u}}}\right)$, and hence, it is dually closed and dually embedded in $\left(\widetilde{G}, \tau_{\widetilde{\mathbf{u}}}\right)$. Now, Lemma 4 of [16] yields $\left\langle\widetilde{\mathbf{u}}_{m}\right\rangle=\operatorname{ker}(\widetilde{\alpha})$, and $\left(\left\langle\widetilde{\mathbf{u}}_{m}\right\rangle,\left.\tau_{\widetilde{\mathbf{u}}}\right|_{\left\langle\widetilde{\mathbf{u}}_{m}\right\rangle}\right)$ is MinAP.

Step 3. Set $\mathbf{v}=\left\{v_{n}\right\}_{n \in \omega}$, where $v_{n}=u_{n+m}$ for every $n \in \omega$. Clearly, $\mathbf{v}$ is a $T$-sequence in $G$ characterizing $H, \tau_{\mathbf{u}}=\tau_{\mathbf{v}}$ and $K_{\mathbf{u}} \subseteq K_{\mathbf{v}}$. Let $t: X \rightarrow X / K_{\mathbf{v}}$ and $r: X / K_{\mathbf{u}} \rightarrow X / K_{\mathbf{v}}$ be the quotient maps. Analogously to Step 1 and the proof of Lemma 23, the sequence $\widetilde{\mathbf{v}}=\left\{\widetilde{v}_{n}\right\}_{n \in \omega}$ is a $T$-sequence in $\widehat{X / K_{\mathbf{v}}}$, which characterizes $H / K_{\mathbf{v}}$ in $X / K_{\mathbf{v}}$, where $v_{n}=\widetilde{v}_{n} \circ t$. Since $t=r \circ q$, we have:

$$
v_{n}=\widetilde{v}_{n} \circ t=t^{\wedge}\left(\widetilde{v}_{n}\right)=q^{\wedge}\left(r^{\wedge}\left(\widetilde{v}_{n}\right)\right)
$$

where $t^{\wedge}, r^{\wedge}$ and $q^{\wedge}$ are the adjoint homomorphisms to $t, r$ and $q$, respectively.

Since $q^{\wedge}$ and $r^{\wedge}$ are embeddings, we have $r^{\wedge}\left(\widetilde{v}_{n}\right)=\widetilde{u}_{n+m}$. In particular, $\langle\mathbf{v}\rangle \cong\langle\widetilde{\mathbf{v}}\rangle \cong\left\langle\widetilde{\mathbf{u}}_{m}\right\rangle$ and :

$$
\left(\left\langle\widetilde{\mathbf{u}}_{m}\right\rangle,\left.\tau_{\widetilde{\mathbf{u}}}\right|_{\left\langle\widetilde{\mathbf{u}}_{m}\right\rangle}\right)=\left(\left\langle\widetilde{\mathbf{u}}_{m}\right\rangle,\left.\tau_{\widetilde{\mathbf{u}}_{m}}\right|_{\left\langle\widetilde{\mathbf{u}}_{m}\right\rangle}\right) \cong\left(\langle\widetilde{\mathbf{v}}\rangle,\left.\tau_{\widetilde{\mathbf{v}}}\right|_{\langle\widetilde{\mathbf{v}}\rangle}\right) \cong\left(\langle\mathbf{v}\rangle, \tau_{\mathbf{v}} \mid\langle\mathbf{v}\rangle\right)
$$

By Step 2, $\left(\left\langle\widetilde{\mathbf{u}}_{m}\right\rangle,\left.\tau_{\widetilde{\mathbf{u}}_{m}}\right|_{\left\langle\widetilde{\mathbf{u}}_{m}\right\rangle}\right)$ is MinAP. Hence, $\left(\langle\mathbf{v}\rangle,\left.\tau_{\mathbf{v}}\right|_{\langle\mathbf{v}\rangle}\right)$ is MinAP, as well.

Step 4. By the second exact sequence in Equation (2) applying to $\mathbf{v}$, Fact 8, and since $\left(\langle\mathbf{v}\rangle, \tau_{\mathbf{v}} \mid\langle\mathbf{v}\rangle\right)$ is MinAP (by Step 3), we have $H=s_{\mathbf{v}}(X)=\left(G, \tau_{\mathbf{v}}\right)^{\wedge}=(G /\langle\mathbf{v}\rangle)^{\wedge}=\langle\mathbf{v}\rangle^{\perp}$ algebraically. Thus, $H^{\perp}=\langle\mathbf{v}\rangle$, and hence, $H^{\perp}$ admits a MinAP group topology generated by the $T$-sequence $\mathbf{v}$. 
$(2) \Rightarrow(1)$ : Since $H$ is a $G_{\delta}$-subgroup of $X, H$ is closed by [13] (Proposition 2.4) and $X / H$ is metrizable (due to the well-known fact that a compact group of countable pseudo-character is metrizable). Hence, $H^{\perp}=(X / H)^{\wedge}$ is countable. Since $H^{\perp}$ admits a MinAP group topology, $H^{\perp}$ must be countably infinite. By Theorem 3.8 of [9], $H^{\perp}$ admits a MinAP group topology generated by a $T$-sequence $\widetilde{\mathbf{u}}=\left\{\widetilde{u}_{n}\right\}_{n \in \omega}$. By Fact 8 , this means that $s \widetilde{\mathbf{u}}(X / H)=\{0\}$. Let $q: X \rightarrow X / H$ be the quotient map. Set $u_{n}=\widetilde{u}_{n} \circ q=q^{\wedge}\left(\widetilde{u}_{n}\right)$. Since $q^{\wedge}$ is injective, $\mathbf{u}$ is a $T$-sequence in $\widehat{X}$ by Lemma 20. We have to show that $H=s_{\mathbf{u}}(X)$. By definition, $x \in s_{\mathbf{u}}(X)$ if and only if:

$$
\left(u_{n}, x\right)=\left(\widetilde{u}_{n}, q(x)\right) \rightarrow 1 \Leftrightarrow q(x) \in s_{\widetilde{\mathbf{u}}}(X / H) \Leftrightarrow q(x)=0 \Leftrightarrow x \in H .
$$

(2) $\Leftrightarrow(3)$ follows from Theorem 3.8 of [9]. The theorem is proven.

Proof of Theorem 14. (1) $\Rightarrow(2)$ : Suppose for a contradiction that $X$ is not connected. Then, by [24] (24.25), the dual group $G=X^{\wedge}$ has a non-zero element $g$ of finite order. Then, the subgroup $H:=\langle g\rangle^{\perp}$ of $X$ has finite index. Hence, $H$ is an open subgroup of $X$. Thus, $H$ is not $T$-characterized by Corollary 13. This contradiction shows that $X$ must be connected.

$(2) \Rightarrow(1)$ : Let $H$ be a proper $G_{\delta}$-subgroup of $X$. Then, $H$ is closed by [13] (Proposition 2.4), and $X / H$ is connected and non-zero. Hence, $H^{\perp} \cong(X / H)^{\wedge}$ is countably infinite and torsion free by [24] (24.25). Thus, $H^{\perp}$ has infinite exponent. Therefore, by Theorem 10, $H$ is $T$-characterized.

The next proposition is a simple corollary of Theorem B in [13].

Proposition 27. The closure $\bar{H}$ of a characterized (in particular, T-characterized) subgroup $H$ of a compact Abelian group $X$ is a characterized subgroup of $X$.

Proof. By Theorem B of [13], $H$ contains a compact $G_{\delta}$-subgroup $K$ of $X$. Then, $\bar{H}$ is also a $G_{\delta}$-subgroup of $X$. Thus, $\bar{H}$ is a characterized subgroup of $X$ by Theorem B of [13].

In general, we cannot assert that the closure $\bar{H}$ of a $T$-characterized subgroup $H$ of a compact Abelian group $X$ is also $T$-characterized, as the next example shows.

Example 1. Let $X=\mathbb{Z}(2) \times \mathbb{T}$ and $G=\widehat{X}=\mathbb{Z}(2) \times \mathbb{Z}$. It is known (see the end of (1) in [7]) that there is a $T$-sequence $\mathbf{u}$ in $G$, such that the von Neumann radical $\mathbf{n}\left(G, \tau_{\mathbf{u}}\right)$ of $\left(G, \tau_{\mathbf{u}}\right)$ is $\mathbb{Z}(2) \times\{0\}$, the subgroup $H:=s_{\mathbf{u}}(X)$ is countable and $\bar{H}=\{0\} \times \mathbb{T}$. Therefore, the closure $\bar{H}$ of the countable $T$-characterized subgroup $H$ of $X$ is open. Thus, $\bar{H}$ is not $T$-characterized by Corollary 13.

We do not know the answers to the following questions:

Problem 28. Let $H$ be a characterized subgroup of a compact Abelian group $X$, such that its closure $\bar{H}$ is $T$-characterized. Is $H$ a $T$-characterized subgroup of $X$ ?

Problem 29. Does there exists a metrizable Abelian compact group that has a countable non-T-characterized subgroup? 


\section{The Proofs of Theorems 16 and 18}

Recall that a Borel subgroup $H$ of a Polish group $X$ is called polishable if there exists a Polish group topology $\tau$ on $H$, such that the inclusion map $i:(H, \tau) \rightarrow X$ is continuous. Let $H$ be a $T$-characterized subgroup of a compact metrizable Abelian group $X$ by a $T$-sequence $\mathbf{u}=\left\{u_{n}\right\}_{n \in \omega}$. Then, by [16] (Theorem 1), $H$ is polishable by the metric:

$$
\rho(x, y)=d(x, y)+\sup \left\{\left|\left(u_{n}, x\right)-\left(u_{n}, y\right)\right|, n \in \omega\right\}
$$

where $d$ is the initial metric on $X$. Clearly, the topology generated by the metric $\rho$ on $H$ is finer than the induced one from $X$.

To prove Theorem 16 we need the following three lemmas.

For a real number $x$, we write $[x]$ for the integral part of $x$ and $\|x\|$ for the distance from $x$ to the nearest integer. We also use the following inequality proven in [25]:

$$
\pi|\varphi| \leq\left|1-e^{2 \pi i \varphi}\right| \leq 2 \pi|\varphi|, \quad \varphi \in\left[-\frac{1}{2}, \frac{1}{2}\right)
$$

Lemma 30. Let $\left\{a_{n}\right\}_{n \in \omega} \subset \mathbb{N}$ be such that $a_{n} \rightarrow \infty$ and $a_{n} \geq 2, n \in \omega$. Set $u_{n}=\prod_{k \leq n} a_{n}$ for every $n \in \omega$. Then, $\mathbf{u}=\left\{u_{n}\right\}_{n \in \omega}$ is a T-sequence in $X=\mathbb{T}$, and the T-characterized subgroup $H=s_{\mathbf{u}}(\mathbb{T})$ of $\mathbb{T}$ is a dense non- $F_{\sigma}$-subset of $\mathbb{T}$.

Proof. We consider the circle group $\mathbb{T}$ as $\mathbb{R} / \mathbb{Z}$ and write it additively. Therefore, $d(0, x)=\|x\|$ for every $x \in \mathbb{T}$. Recall that every $x \in \mathbb{T}$ has the unique representation in the form:

$$
x=\sum_{n=0}^{\infty} \frac{c_{n}}{u_{n}}
$$

where $0 \leq c_{n}<a_{n}$ and $c_{n} \neq a_{n}-1$ for infinitely many indices $n$.

It is known [26] (see also (12) in the proof of Lemma 1 of [25]) that $x$ with representation Equation (6) belongs to $H$ if and only if:

$$
\lim _{n \rightarrow \infty} \frac{c_{n}}{a_{n}}(\bmod 1)=0 .
$$

Hence, $H$ is a dense subgroup of $\mathbb{T}$. Thus, $\mathbf{u}$ is even a $T B$-sequence in $\mathbb{Z}$ by Fact 1 .

We have to show that $H$ is not an $F_{\sigma}$-subset of $\mathbb{T}$. Suppose for a contradiction that $H$ is an $F_{\sigma}$-subset of $\mathbb{T}$. Then, $H=\cup_{n \in \mathbb{N}} F_{n}$, where $F_{n}$ is a compact subset of $\mathbb{T}$ for every $n \in \mathbb{N}$. Since $H$ is a subgroup of $\mathbb{T}$, without loss of generality, we can assume that $F_{n}-F_{n} \subseteq F_{n+1}$. Since all $F_{n}$ are closed in $(H, \rho)$, as well, the Baire theorem implies that there are $0<\varepsilon<0.1$ and $m \in \mathbb{N}$, such that $F_{m} \supseteq\{x: \rho(0, x) \leq \varepsilon\}$.

Fix arbitrarily $l>0$, such that $\frac{2}{u_{l-1}}<\frac{\varepsilon}{20}$. For every natural number $k>l$, set:

$$
x_{k}:=\sum_{n=l}^{k} \frac{1}{u_{n}} \cdot\left[\frac{\left(a_{n}-1\right) \varepsilon}{20}\right] .
$$

Then, for every $k>l$, we have:

$$
x_{k}=\sum_{n=l}^{k} \frac{1}{u_{n}} \cdot\left[\frac{\left(a_{n}-1\right) \varepsilon}{20}\right]<\sum_{n=l}^{k} \frac{1}{u_{n-1}} \cdot \frac{\varepsilon}{20}<\frac{1}{u_{l-1}} \sum_{n=0}^{k-l} \frac{1}{2^{n}}<\frac{2}{u_{l-1}}<\frac{\varepsilon}{20}<\frac{1}{2} .
$$


This inequality and Equation (5) imply that:

$$
d\left(0, x_{k}\right)=\left\|x_{k}\right\|=x_{k}<\frac{\varepsilon}{20}, \text { for every } k>l .
$$

For every $s \in \omega$ and every natural number $k>l$, we estimate $\left|1-\left(u_{s}, x_{k}\right)\right|$ as follows.

Case 1. Let $s<k$. Set $q=\max \{s+1, l\}$. By the definition of $x_{k}$, we have:

$$
\begin{aligned}
2 \pi\left[\left(u_{s} \cdot x_{k}\right)(\bmod 1)\right] & =2 \pi\left[u_{s} \sum_{n=l}^{k} \frac{1}{u_{n}} \cdot\left[\frac{\left(a_{n}-1\right) \varepsilon}{20}\right](\bmod 1)\right]<2 \pi \sum_{n=q}^{k} \frac{u_{s}}{u_{n}} \cdot \frac{\left(a_{n}-1\right) \varepsilon}{20} \\
& <\frac{\pi \varepsilon}{10}\left(1+\frac{1}{a_{s+1}}+\frac{1}{a_{s+1} a_{s+2}}+\frac{1}{a_{s+1} a_{s+2} a_{s+3}}+\ldots\right) \\
& <\frac{\pi \varepsilon}{10}\left(1+\frac{1}{2}+\frac{1}{2^{2}}+\frac{1}{2^{3}}+\ldots\right)=\frac{\pi \varepsilon}{10} \cdot 2<\frac{2 \varepsilon}{3}<\frac{1}{2} .
\end{aligned}
$$

This inequality and Equation (5) imply:

$$
\left|1-\left(u_{s}, x_{k}\right)\right|=\left|1-\exp \left\{2 \pi i \cdot\left[\left(u_{s} \cdot x_{k}\right)(\bmod 1)\right]\right\}\right|<\frac{2 \varepsilon}{3} .
$$

Case 2. Let $s \geq k$. By the definition of $x_{k}$, we have:

$$
\left|1-\left(u_{s}, x_{k}\right)\right|=0
$$

In particular, Equation (10) implies that $x_{k} \in H$ for every $k>l$.

Now, for every $k>l$, Equations (4) and (8)-(10) imply:

$$
\rho\left(0, x_{k}\right)<\frac{\varepsilon}{20}+\frac{2 \varepsilon}{3}<\varepsilon
$$

Thus, $x_{k} \in F_{m}$ for every natural number $k>l$. Clearly,

$$
x_{k} \rightarrow x:=\sum_{n=l}^{\infty} \frac{1}{u_{n}} \cdot\left[\frac{\left(a_{n}-1\right) \varepsilon}{20}\right] \quad \text { in } \mathbb{T} .
$$

Since $F_{m}$ is a compact subset of $\mathbb{T}$, we have $x \in F_{m}$. Hence, $x \in H$. On the other hand, we have:

$$
\lim _{n \rightarrow \infty} \frac{1}{a_{n}} \cdot\left[\frac{\left(a_{n}-1\right) \varepsilon}{20}\right](\bmod 1)=\frac{\varepsilon}{20} \neq 0 .
$$

Therefore, Equation (7) implies that $x \notin H$. This contradiction shows that $H=s_{\mathbf{u}}(\mathbb{T})$ is not an $F_{\sigma}$-subset of $\mathbb{T}$.

For a prime number $p$, the group $\mathbb{Z}\left(p^{\infty}\right)$ is regarded as the collection of fractions $m / p^{n} \in[0,1)$. Let $\Delta_{p}$ be the compact group of $p$-adic integers. It is well known that $\widehat{\Delta_{p}}=\mathbb{Z}\left(p^{\infty}\right)$.

Lemma 31. Let $X=\Delta_{p}$. For an increasing sequence of natural numbers $0<n_{0}<n_{1}<\ldots$, such that $n_{k+1}-n_{k} \rightarrow \infty$, set:

$$
u_{k}=\frac{1}{p^{n_{k}+1}} \in \mathbb{Z}\left(p^{\infty}\right) .
$$

Then, the sequence $\mathbf{u}=\left\{u_{k}\right\}_{k \in \omega}$ is a T-sequence in $\mathbb{Z}\left(p^{\infty}\right)$, and the T-characterized subgroup $H=s_{\mathbf{u}}\left(\Delta_{p}\right)$ is a dense non- $F_{\sigma}$-subset of $\Delta_{p}$. 
Proof. Let $\omega=\left(a_{n}\right)_{n \in \omega} \in \Delta_{p}$, where $0 \leq a_{n}<p$ for every $n \in \omega$. Recall that, for every $k \in \omega$, [24] (25.2) implies:

$$
\left(u_{k}, \omega\right)=\exp \left\{\frac{2 \pi i}{p^{n_{k}+1}}\left(a_{0}+p a_{1}+\cdots+p^{n_{k}} a_{n_{k}}\right)\right\} .
$$

Further, by [24] (10.4), if $\omega \neq 0$, then $d(0, \omega)=2^{-n}$, where $n$ is the minimal index, such that $a_{n} \neq 0$. Following [27] (2.2), for every $\omega=\left(a_{n}\right) \in \Delta_{p}$ and every natural number $k>1$, set:

$$
m_{k}=m_{k}(\omega)=\max \left\{j_{k}, n_{k-1}\right\}
$$

where:

$$
j_{k}=n_{k} \text { if } 0<a_{n_{k}}<p-1
$$

and otherwise:

$$
j_{k}=\min \left\{j: \text { either } a_{s}=0 \text { for } j<s \leq n_{k}, \text { or } a_{s}=p-1 \text { for } j<s \leq n_{k}\right\}
$$

In [27] (2.2), it is shown that:

$$
\omega \in s_{\mathbf{u}}\left(\Delta_{p}\right) \text { if and only if } n_{k}-m_{k} \rightarrow \infty
$$

Therefore, $H:=s_{\mathbf{u}}\left(\Delta_{p}\right)$ contains the identity $\mathbf{1}=(1,0,0, \ldots)$ of $\Delta_{p}$. By [24] (Remark 10.6), $\langle\mathbf{1}\rangle$ is dense in $\Delta_{p}$. Hence, $H$ is dense in $\Delta_{p}$, as well. Now, Fact 1 implies that $\mathbf{u}$ is a $T$-sequence in $\mathbb{Z}\left(p^{\infty}\right)$.

We have to show that $H$ is not an $F_{\sigma}$-subset of $\Delta_{p}$. Suppose for a contradiction that $H=\cup_{n \in \mathbb{N}} F_{n}$ is an $F_{\sigma}$-subset of $\Delta_{p}$, where $F_{n}$ is a compact subset of $\Delta_{p}$ for every $n \in \mathbb{N}$. Since $H$ is a subgroup of $\Delta_{p}$, without loss of generality, we can assume that $F_{n}-F_{n} \subseteq F_{n+1}$. Since all $F_{n}$ are closed in $(H, \rho)$, as well, the Baire theorem implies that there are $0<\varepsilon<0.1$ and $m \in \mathbb{N}$, such that $F_{m} \supseteq\{x: \rho(0, x) \leq \varepsilon\}$.

Fix a natural number $s$, such that $\frac{1}{2^{s}}<\frac{\varepsilon}{20}$. Choose a natural number $l>s$, such that, for every natural number $w \geq l$, we have:

$$
n_{w+1}-n_{w}>s
$$

For every $r \in \mathbb{N}$, set:

$$
\omega_{r}:=\left(a_{n}^{r}\right), \text { where } a_{n}^{r}=\left\{\begin{array}{l}
1, \text { if } n=n_{l+i}-s \text { for some } 1 \leq i \leq r, \\
0, \text { otherwise. }
\end{array}\right.
$$

Then, for every $r \in \mathbb{N}$, Equation (13) implies that $\omega_{r}$ is well defined and:

$$
d\left(0, \omega_{r}\right)=\frac{1}{2^{n_{l+1}-s}}<\frac{1}{2^{n_{l}}} \leq \frac{1}{2^{l}}<\frac{1}{2^{s}}<\frac{\varepsilon}{20} .
$$

Note that:

$$
1+p+\cdots+p^{k}=\frac{p^{k+1}-1}{p-1}<p^{k+1} .
$$

For every $k \in \omega$ and every $r \in \mathbb{N}$, we estimate $\left|1-\left(u_{k}, \omega_{r}\right)\right|$ as follows.

Case 1. Let $k \leq l$. By Equations (11) and (13) and the definition of $\omega_{r}$, we have:

$$
\left|1-\left(u_{k}, \omega_{r}\right)\right|=0
$$


Case 2. Let $l<k \leq l+r$. Then, Equation (15) yields:

$$
\frac{2 \pi}{p^{n_{k}+1}}\left|p^{n_{l+1}-s}+\cdots+p^{n_{k}-s}\right|<\frac{2 \pi}{p^{n_{k}+1}} \cdot p^{n_{k}-s+1}=\frac{2 \pi}{p^{s}} \leq \frac{2 \pi}{2^{s}}<\frac{\varepsilon}{2}<\frac{1}{2} .
$$

This inequality and the inequality Equations (5) and (11) imply:

$$
\left|1-\left(u_{k}, \omega_{r}\right)\right|=\left|1-\exp \left\{\frac{2 \pi i}{p^{n_{k}+1}}\left(p^{n_{l+1}-s}+\cdots+p^{n_{k}-s}\right)\right\}\right|<\frac{\varepsilon}{2} .
$$

Case 3. Let $l+r<k$. By Equation (15), we have:

$$
\begin{aligned}
\frac{2 \pi}{p^{n_{k}+1}}\left|p^{n_{l+1}-s}+\cdots+p^{n_{l+r}-s}\right| & <\frac{2 \pi}{p^{n_{k}+1}} \cdot p^{n_{l+r}-s+1} \\
& <\frac{2 \pi}{p^{n_{k}+1}} \cdot p^{n_{k}-s+1}=\frac{2 \pi}{p^{s}} \leq \frac{2 \pi}{2^{s}}<\frac{\varepsilon}{2} .
\end{aligned}
$$

These inequalities, Equations (5) and (11) immediately yield:

$$
\left|1-\left(u_{k}, \omega_{r}\right)\right|=\left|1-\exp \left\{\frac{2 \pi i}{p^{n_{k}+1}}\left(p^{n_{l+1}-s}+\cdots+p^{n_{l+r}-s}\right)\right\}\right|<\frac{\varepsilon}{2},
$$

and:

$$
\left|1-\left(u_{k}, \omega_{r}\right)\right|<\frac{2 \pi}{p^{n_{k}+1}} \cdot p^{n_{l+r}-s+1} \rightarrow 0, \text { as } k \rightarrow \infty .
$$

Therefore, Equation (19) implies that $\omega_{r} \in H$ for every $r \in \mathbb{N}$.

For every $r \in \mathbb{N}$, by Equations (4), (14) and (16)-(18), we have:

$$
\rho\left(0, \omega_{r}\right)=d\left(0, \omega_{r}\right)+\sup \left\{\left|1-\left(u_{k}, \omega_{r}\right)\right|, k \in \omega\right\}<\frac{\varepsilon}{20}+\frac{\varepsilon}{2}<\varepsilon .
$$

Thus, $\omega_{r} \in F_{m}$ for every $r \in \mathbb{N}$. Evidently,

$$
\omega_{r} \rightarrow \widetilde{\omega}=\left(\widetilde{a}_{n}\right) \text { in } \Delta_{p}, \text { where } \widetilde{a}_{n}=\left\{\begin{array}{l}
1, \text { if } n=n_{l+i}-s \text { for some } i \in \mathbb{N}, \\
0, \text { otherwise }
\end{array}\right.
$$

Since $F_{m}$ is a compact subset of $\Delta_{p}$, we have $\widetilde{\omega} \in F_{m}$. Hence, $\widetilde{\omega} \in H$. On the other hand, it is clear that $m_{k}(\widetilde{\omega})=n_{k}-s$ for every $k \geq l+1$. Thus, for every $k \geq l+1, n_{k}-m_{k}(\widetilde{\omega})=s \not \rightarrow \infty$. Now, Equation (12) implies that $\widetilde{\omega} \notin H$. This contradiction shows that $H$ is not an $F_{\sigma}$-subset of $\Delta_{p}$.

Lemma 32. Let $X=\prod_{n \in \omega} \mathbb{Z}\left(b_{n}\right)$, where $1<b_{0}<b_{1}<\ldots$ and $G:=\widehat{X}=\bigoplus_{n \in \omega} \mathbb{Z}\left(b_{n}\right)$. Set $\mathbf{u}=\left\{u_{n}\right\}_{n \in \omega}$, where $u_{n}=1 \in \mathbb{Z}\left(b_{n}\right)^{\wedge} \subset G$ for every $n \in \omega$. Then, $\mathbf{u}$ is a T-sequence in $G$, and the T-characterized subgroup $H=s_{\mathbf{u}}(X)$ is a dense non-F-subset of $X$.

Proof. Set $H:=s_{\mathbf{u}}(X)$. In [27] (2.3), it is shown that:

$$
\omega=\left(a_{n}\right) \in s_{\mathbf{u}}(X) \text { if and only if }\left\|\frac{a_{n}}{b_{n}}\right\| \rightarrow 0 .
$$

Therefore, $\bigoplus_{n \in \omega} \mathbb{Z}\left(b_{n}\right) \subseteq H$. Thus, $H$ is dense in $X$. Now, Fact 1 implies that $\mathbf{u}$ is a $T$-sequence in $G$. 
We have to show that $H$ is not an $F_{\sigma}$-subset of $X$. Suppose for a contradiction that $H=\cup_{n \in \mathbb{N}} F_{n}$ is an $F_{\sigma}$-subset of $X$, where $F_{n}$ is a compact subset of $X$ for every $n \in \mathbb{N}$. Since $H$ is a subgroup of $X$, without loss of generality, we can assume that $F_{n}-F_{n} \subseteq F_{n+1}$. Since all $F_{n}$ are closed in $(H, \rho)$, as well, the Baire theorem yields that there are $0<\varepsilon<0.1$ and $m \in \mathbb{N}$, such that $F_{m} \supseteq\{\omega \in X: \rho(0, \omega) \leq \varepsilon\}$.

Note that $d(0, \omega)=2^{-l}$, where $0 \neq \omega=\left(a_{n}\right)_{n \in \omega} \in X$ and $l$ is the minimal index, such that $a_{l} \neq 0$. Choose $l$, such that $2^{-l}<\varepsilon / 3$. For every natural number $k>l$, set:

$$
\omega_{k}:=\left(a_{n}^{k}\right), \text { where } a_{n}^{k}=\left\{\begin{array}{c}
{\left[\frac{\varepsilon b_{n}}{20}\right], \text { for every } n \text { such that } l \leq n \leq k,} \\
0, \text { if either } 1 \leq n<l \text { or } k<n .
\end{array}\right.
$$

Since $\left(u_{n}, \omega_{k}\right)=1$ for every $n>k$, we obtain that $\omega_{k} \in H$ for every $k>l$. For every $n \in \omega$, we have:

$$
2 \pi \cdot \frac{1}{b_{n}}\left[\frac{\varepsilon b_{n}}{20}\right]<\frac{2 \pi \varepsilon}{20}<\varepsilon<\frac{1}{2} .
$$

This inequality and the inequality Equations (4) and (5) imply:

$$
\begin{aligned}
\rho\left(0, \omega_{k}\right) & =d\left(0, \omega_{k}\right)+\sup \left\{\left|1-\left(u_{n}, \omega_{k}\right)\right|, n \in \omega\right\} \\
& \leq \frac{1}{2^{l}}+\max \left\{\left|1-\exp \left\{2 \pi i \frac{1}{b_{n}}\left[\frac{\varepsilon b_{n}}{20}\right]\right\}\right|, l \leq n \leq k\right\} \\
& \leq \frac{\varepsilon}{3}+2 \pi \cdot \max \left\{\frac{1}{b_{n}}\left[\frac{\varepsilon b_{n}}{20}\right], l \leq n \leq k\right\}<\frac{\varepsilon}{3}+\frac{2 \pi \varepsilon}{20}<\varepsilon .
\end{aligned}
$$

Thus, $\omega_{k} \in F_{m}$ for every natural number $k>l$. Evidently,

$$
\omega_{k} \rightarrow \widetilde{\omega}=\left(\widetilde{a}_{n}\right)_{n \in \omega} \text { in } X, \text { where } \widetilde{a}_{n}=\left\{\begin{array}{r}
0, \text { if } 0 \leq n<l, \\
{\left[\frac{\varepsilon b_{n}}{20}\right], \text { if } l \leq n .}
\end{array}\right.
$$

Since $F_{m}$ is a compact subset of $X$, we have $\widetilde{\omega} \in F_{m}$. Hence, $\widetilde{\omega} \in H$. On the other hand, since $b_{n} \rightarrow \infty$, we have:

$$
\lim _{n \rightarrow \infty}\left\|\frac{\widetilde{a}_{n}}{b_{n}}\right\|=\lim _{n \rightarrow \infty} \frac{1}{b_{n}}\left[\frac{\varepsilon b_{n}}{20}\right]=\frac{\varepsilon}{20} \neq 0 .
$$

Thus, $\widetilde{\omega} \notin H$ by Equation (20). This contradiction shows that $H$ is not an $F_{\sigma}$-subset of $X$.

Now, we are in a position to prove Theorems 16 and 18.

Proof of Theorem 16. Let $X$ be a compact Abelian group of infinite exponent. Then, $G:=\widehat{X}$ also has infinite exponent. It is well-known that $G$ contains a countably-infinite subgroup $S$ of one of the following form:

(a) $S \cong \mathbb{Z}$;

(b) $S \cong \mathbb{Z}\left(p^{\infty}\right)$;

(c) $S \cong \bigoplus_{n \in \omega} \mathbb{Z}\left(b_{n}\right)$, where $1<b_{0}<b_{1}<\ldots$.

Fix such a subgroup $S$. Set $K=S^{\perp}$ and $Y=X / K \cong S_{d}^{\wedge}$, where $S_{d}$ denotes the group $S$ endowed with the discrete topology. Since $S$ is countable, $Y$ is metrizable. Hence, $\{0\}$ is a $G_{\delta}$-subgroup of 
$Y$. Thus, $K$ is a $G_{\delta}$-subgroup of $X$. Let $q: X \rightarrow Y$ be the quotient map. By Lemmas 30-32, the compact group $Y$ has a dense $T$-characterized subgroup $\widetilde{H}$, which is not an $F_{\sigma}$-subset of $Y$. Lemma 24 implies that $H:=q^{-1}(\widetilde{H})$ is a dense $T$-characterized subgroup of $X$. Since the continuous image of an $F_{\sigma}$-subset of a compact group is an $F_{\sigma}$-subset, as well, we obtain that $H$ is not an $F_{\sigma}$-subset of $X$. Thus, the subgroup $H$ of $X$ is $T$-characterized, but it is not an $F_{\sigma}$-subset of $X$. The theorem is proven.

Proof of Theorem 18. (1) Follows from Fact 5.

(2) By Lemma 3.6 in [13], every infinite compact Abelian group $X$ contains a dense characterized subgroup $H$. By Fact $1, H$ is $T$-characterized. Since every $G_{\delta}$-subgroup of $X$ is closed in $X$ by Proposition 2.4 of [13], $H$ is not a $G_{\delta}$-subgroup of $X$.

(3) Follows from Theorem 14 and the aforementioned Proposition 2.4 of [13].

(4) Follows from Fact 5.

(5) Follows from Corollary 17.

It is trivial that $\operatorname{Char}_{T}(X) \subseteq \operatorname{Char}(X)$ for every compact Abelian group $X$. For the circle group $\mathbb{T}$, we have:

Proposition 33. $\operatorname{Char}_{T}(\mathbb{T})=\operatorname{Char}(\mathbb{T})$.

Proof. We have to show only that $\operatorname{Char}(\mathbb{T}) \subseteq \operatorname{Char}_{T}(\mathbb{T})$. Let $H=s_{\mathbf{u}}(\mathbb{T}) \in \operatorname{Char}(\mathbb{T})$ for some sequence $\mathbf{u}$ in $\mathbb{Z}$.

If $H$ is infinite, then $H$ is dense in $\mathbb{T}$. Therefore, $\mathbf{u}$ is a $T$-sequence in $\mathbb{Z}$ by Fact 1 . Thus, $H \in$ $\operatorname{Char}_{T}(\mathbb{T})$.

If $H$ is finite, then $H$ is closed in $\mathbb{T}$. Clearly, $H^{\perp}$ has infinite exponent. Thus, $H \in \operatorname{Char}_{T}(\mathbb{T})$ by Theorem 10.

Note that, if a compact Abelian group $X$ satisfies the equality $\operatorname{Char}_{T}(X)=\operatorname{Char}(X)$, then $X$ is connected by Fact 3 and Theorem 14. This fact and Proposition 33 justify the next problem:

Problem 34. Does there exists a connected compact Abelian group $X$, such that $\operatorname{Char}_{T}(X) \neq$ $\operatorname{Char}(X)$ ? Is it true that $\operatorname{Char}_{T}(X)=\operatorname{Char}(X)$ if and only if $X$ is connected?

For a compact Abelian group $X$, the set of all subgroups of $X$ that are both $F_{\sigma \delta^{-}}$and $G_{\delta \sigma^{-}}$-subsets of $X$ we denote by $\mathrm{S}_{3}^{0}(X)$. To complete the study of the Borel hierarchy of $(T$-)characterized subgroups of $X$, we have to answer the next question.

Problem 35. Describe compact Abelian groups $X$ of infinite exponent for which Char $(X) \subseteq \mathrm{S} \Delta_{3}^{0}(X)$. For which compact Abelian groups $X$ of infinite exponent there exists a T-characterized subgroup $H$ that does not belong to $\mathrm{S} \Delta_{3}^{0}(X)$ ? 


\section{4. $\mathfrak{g}_{T}$-Closed and $\mathfrak{g}_{T}$-Dense Subgroups of Compact Abelian Groups}

The following closure operator $\mathfrak{g}$ of the category of Abelian topological groups is defined in [11]. Let $X$ be an Abelian topological group and $H$ its arbitrary subgroup. The closure operator $\mathfrak{g}=\mathfrak{g}_{X}$ is defined as follows:

$$
\mathfrak{g}_{X}(H):=\bigcap_{\mathbf{u} \in \widehat{X}^{\mathbb{N}}}\left\{s_{\mathbf{u}}(X): H \leq s_{\mathbf{u}}(X)\right\},
$$

and we say that $H$ is $\mathfrak{g}$-closed if $H=\mathfrak{g}(H)$, and $H$ is $\mathfrak{g}$-dense if $\mathfrak{g}(H)=X$.

The set of all $T$-sequences in the dual group $\widehat{X}$ of a compact Abelian group $X$ we denote by $\mathcal{T}_{s}(\widehat{X})$. Clearly, $\mathcal{T}_{s}(\widehat{X}) \varsubsetneqq \widehat{X}^{\mathbb{N}}$. Let $H$ be a subgroup of $X$. In analogy to the closure operator $\mathfrak{g}, \mathfrak{g}$-closure and $\mathfrak{g}$-density, the operator $\mathfrak{g}_{T}$ is defined as follows:

$$
\mathfrak{g}_{T}(H):=\bigcap_{\mathbf{u} \in \mathcal{T}_{s}(\widehat{X})}\left\{s_{\mathbf{u}}(X): H \leq s_{\mathbf{u}}(X)\right\},
$$

and we say that $H$ is $\mathfrak{g}_{T}$-closed if $H=\mathfrak{g}_{T}(H)$, and $H$ is $\mathfrak{g}_{T}$-dense if $\mathfrak{g}_{T}(H)=X$.

In this section, we study some properties of $\mathfrak{g}_{T}$-closed and $\mathfrak{g}_{T}$-dense subgroups of a compact Abelian group $X$. Note that every $\mathfrak{g}$-dense subgroup of $X$ is dense by Lemma 2.12 of [11], but for $\mathfrak{g}_{T}$-dense subgroups, the situation changes:

Proposition 36. Let $X$ be a compact Abelian group.

(1) If $H$ is a $\mathfrak{g}_{T}$-dense subgroup of $X$, then the closure $\bar{H}$ of $H$ is an open subgroup of $X$.

(2) Every open subgroup of a compact Abelian group $X$ is $\mathfrak{g}_{T}$-dense.

Proof. (1) Suppose for a contradiction that $\bar{H}$ is not open in $X$. Then, $X / \bar{H}$ is an infinite compact group. By Lemma 3.6 of [13], $X / \bar{H}$ has a proper dense characterized subgroup $S$. Fact 1 implies that $S$ is a $T$-characterized subgroup of $X / \bar{H}$. Let $q: X \rightarrow X / \bar{H}$ be the quotient map. Then, Lemma 24 yields that $q^{-1}(S)$ is a $T$-characterized dense subgroup of $X$ containing $H$. Since $q^{-1}(S) \neq X$, we obtain that $H$ is not $\mathfrak{g}_{T}$-dense in $X$, a contradiction.

(2) Let $H$ be an open subgroup of $X$. If $H=X$, the assertion is trivial. Assume that $H$ is a proper subgroup (so $X$ is disconnected). Let $\mathbf{u}$ be an arbitrary $T$-sequence, such that $H \subseteq s_{\mathbf{u}}(X)$. Since $H$ is open, $s_{\mathbf{u}}(X)$ is open, as well. Now, Corollary 13 implies that $s_{\mathbf{u}}(X)=X$. Thus, $H$ is $\mathfrak{g}_{T}$-dense in $X$.

Proposition 36(2) shows that $\mathfrak{g}_{T}$-density may essentially differ from the usual $\mathfrak{g}$-density. In the next theorem, we characterize all compact Abelian groups for which all $\mathfrak{g}_{T}$-dense subgroups are also dense.

Theorem 37. All $\mathfrak{g}_{T}$-dense subgroups of a compact Abelian group $X$ are dense if and only if $X$ is connected.

Proof. Assume that all $\mathfrak{g}_{T}$-dense subgroup of $X$ are dense. Proposition 36(2) implies that $X$ has no open proper subgroups. Thus, $X$ is connected by [24] (7.9).

Conversely, let $X$ be connected and $H$ be a $\mathfrak{g}_{T}$-dense subgroup of $X$. Proposition 36(1) implies that the closure $\bar{H}$ of $H$ is an open subgroup of $X$. Since $X$ is connected, we obtain that $\bar{H}=X$. Thus, $H$ is dense in $X$. 
For $\mathfrak{g}_{T}$-closed subgroups, we have:

Proposition 38. Let $X$ be a compact Abelian group.

(1) Every proper open subgroup $H$ of $X$ is a $\mathfrak{g}$-closed non- $\mathfrak{g}_{T}$-closed subgroup.

(2) If every $\mathfrak{g}$-closed subgroup of $X$ is $\mathfrak{g}_{T}$-closed, then $X$ is connected.

Proof. (1) The subgroup $H$ is $\mathfrak{g}_{T}$-dense in $X$ by Proposition 36. Therefore, $H$ is not $\mathfrak{g}_{T}$-closed. On the other hand, $H$ is $\mathfrak{g}$-closed in $X$ by Theorem A of [13].

(2) Item (1) implies that $X$ has no open subgroups. Thus, $X$ is connected by [24] (7.9).

We do not know whether the converse in Proposition 38(2) holds true:

Problem 39. Let a compact Abelian group $X$ be connected. Is it true that every $\mathfrak{g}$-closed subgroup of $X$ is also $\mathfrak{g}_{T}$-closed?

\section{Conflicts of Interest}

The authors declare no conflict of interest.

\section{References}

1. Graev, M. Free topological groups. Izv. Akad. Nauk SSSR Ser. Mat. 1948, 12, 278-324.

2. Nienhuys, J. Construction of group topologies on Abelian groups. Fund. Math. 1972, 75, 101-116.

3. Protasov, I.V.; Zelenyuk, E.G. Topologies on abelian groups. Math. USSR Izv. 1991, 37, 445-460.

4. Raczkowski, S.U. Totally bounded topological group topologies on the integers. Topol. Appl. 2002, 121, 63-74.

5. Barbieri, G.; Dikranjan, D.; Milan, C.; Weber, H. Answer to Raczkowski’s question on convergent sequences of integers. Topol. Appl. 2003, 132, 89-101.

6. Barbieri, G.; Dikranjan, D.; Milan, C.; Weber, H. Convergent sequences in precompact group topologies. Appl. Gen. Topol. 2005, 6, 149-169.

7. Gabriyelyan, S. Characterization of almost maximally almost-periodic groups. Topol. Appl. 2009, 156, 2214-2219.

8. Gabriyelyan, S. Finitely generated subgroups as a von Neumann radical of an Abelian group. Mat. Stud. 2012, 38, 124-138.

9. Gabriyelyan, S. Bounded subgroups as a von Neumann radical of an Abelian group. Topol. Appl. 2014, 178, 185-199.

10. Protasov, I.V.; Zelenyuk, E.G. Topologies on Groups Determined by Sequences; Monograph Series; Math. Studies VNTL, L'viv, 1999.

11. Dikranjan, D.; Milan, C.; Tonolo, A. A characterization of the MAP abelian groups. J. Pure Appl. Algebra 2005, 197, 23-41.

12. Biró, A.; Deshouillers, J.-M.; Sós, V. Good approximation and characterization of subgroups of $\mathbb{R} / \mathbb{Z}$. Studia Sci. Math. Hungar. 2001, 38, 97-113.

13. Dikranjan, D.; Gabriyelyan, S. On characterized subgroups of compact abelian groups. Topol. Appl. 2013, 160, 2427-2442. 
14. Beiglböck, M.; Steineder, C.; Winkler, R. Sequences and filters of characters characterizing subgroups of compact abelian groups. Topol. Appl. 2006, 153, 1682-1695.

15. Dikranjan, D.; Kunen, K. Characterizing subgroups of compact abelian groups. J. Pure Appl. Algebra 2007, 208, 285-291.

16. Gabriyelyan, S. On T-sequences and characterized subgroups. Topol. Appl. 2010, 157, 2834-2843.

17. Comfort, W.; Raczkowski, S.; Trigos-Arrieta, F.-J. Making group topologies with, and without, convergent sequences. Appl. Gen. Topol. 2006, 7, 109-124.

18. Gabriyelyan, S. On a generalization of Abelian sequential groups. Fund. Math. 2013, 221, 95-127.

19. Noble, N. k-groups and duality. Trans. Amer. Math. Soc. 1970, 151, 551-561.

20. Dikranjan, D.; Gabriyelyan, S.; Tarieladze, V. Characterizing sequences for precompact group topologies. J. Math. Anal. Appl. 2014, 412, 505-519.

21. Gabriyelyan, S. Characterizable groups: Some results and open questions. Topol. Appl. 2012, 159, 2378-2391.

22. Banaszczyk, W.; Chasco, M.; Martin-Peinador, E. Open subgroups and Pontryagin duality. Math. Z. 1994, 215, 195-204.

23. Außenhofer, L. Contributions to the Duality Theory of Abelian Topological Groups and to the Theory of Nuclear Groups. Diss. Math. 1999, 384, 113.

24. Hewitt, E.; Ross, K. Abstract Harmonic Analysis, 2nd ed.; Springer-Verlag: Berlin, Germany, 1979; Volume I.

25. Gabriyelyan, S. Groups of quasi-invariance and the Pontryagin duality. Topol. Appl. 2010, 157, 2786-2802.

26. Aaronson, J.; Nadkarni, M. $L_{\infty}$ eigenvalues and $L_{2}$ spectra of non-singular transformations. Proc. Lond. Math. Soc. 1987, 55, 538-570.

27. Gabriyelyan, S. Reflexive group topologies on Abelian groups. J. Group Theory 2010, 13, 891-901.

(c) 2015 by the author; licensee MDPI, Basel, Switzerland. This article is an open access article distributed under the terms and conditions of the Creative Commons Attribution license (http://creativecommons.org/licenses/by/4.0/). 